\title{
DISCRETE TIMBER ASSEMBLY
}

GILLES RETSIN

THE BARTLETT SCHOOL OF ARCHITECTURE, UCL

\section{Digital Materials}

Using a number of built demonstrators, this paper describes
a computational design and fabrication method for timber assembly, based on the notion of discretenens. This research and Programmable Matter with the architectural field of Prefabrication and Modularity. While these two fields are at opposing ends of the spectrum in terms of scale and of the properties and challenges are transferable.

Neil Gerschenfeld and the Centre for Bits and Atoms at MIT have developed the notion of 'Digital' Materials, Matter Programmable Matter is a wider fold that straddle robotics, computer science, material science and engineering, and focuses on the creation of materials whose properties can be adapted and coded (Gerschenfeld et al. Self-Assembly and Self-Reconfiguring Modult Robotics. Digital materials can be precisely defined as a discrete set of parts, which are reversibly joined with a discrete set of
relative positions and orientations (Cheung, 2012). These can then be assembled into larger scale wholes with for farication' is a process that compiles these discrete whereas analogue fabrication is based on continuous subtraction or deposition of matter. For example, 3D printing and robotic milling are considered analogue fabrication methods.

Some of the challenges digital materials attempt to tackle in robotics and mechanical engineering are highly relevant to architecture and construction. Digital Materials autonomously manufacture functional machines or infrastructures from smaller base units. Architecture in its most basic sense attempts the same: assembling programmable matter is more focused on the active and immediate performance of a whole, its mechanical operation. In architecture, the functionality is concern
with change over a long period of time. Adaptability assembly and disassembly are processes taking place over
weeks, months or years, rather than seconds. Primarily, the transferable aspects from programmable matter and
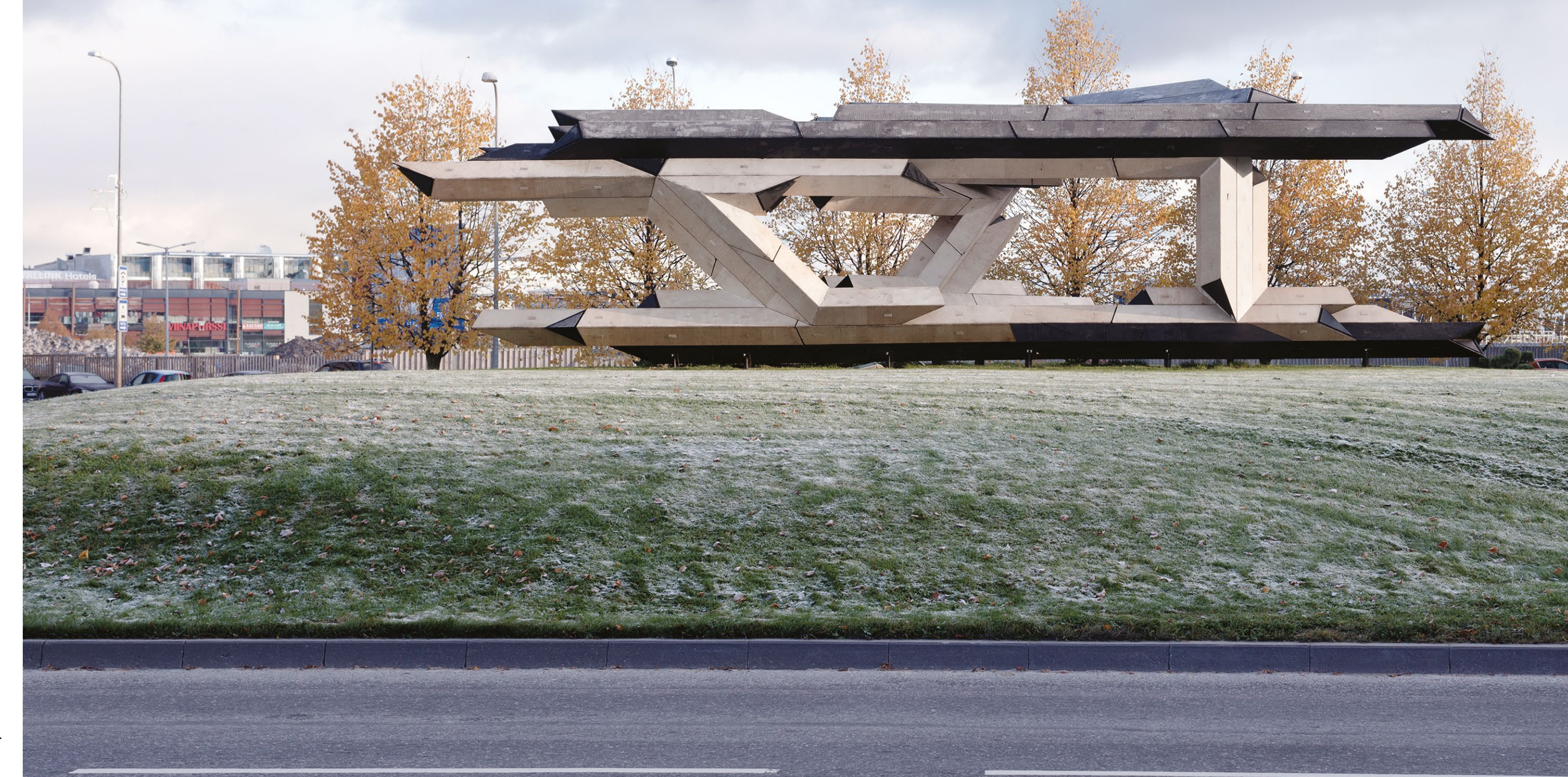
chain, the potential for automation and the concept
of limited modular parts establishing vast variation, ompled me versatility, adaptation and re assembly

over time.

Programmable Matter and Modularity

Digital materials attempt to overcome the discontinuity present in analogue fabrication where unrelated processes
have to be combined. These result in expensive, timehave to be combined. These result in expenswe, timemachine needs its own customised fabrication process (Langford, 2019). Just like robotics and manufacturing, architecture and construction suffers from a similar
analogue syntax with resulting discontinuities, unrela analogue syntax with resulthy disconthinges, unelated of over 7000 different parts and processes which need to be assembled together into a functional whole. This makes construction slow, expensive and difficult to automate. To achieve full automation, every part and process would

In a context of increasing cost of labour and decreasing (a) construction. The construction industry has flat-lined since 1947, whereas manutacturing has radically increased (Mckinsey, 2017). As building is slow and expensive, only a limited number of actors can take the risk to construct. This in turn keeps the market limited and scarce in supply construction in the hands of the few - government large developers. Coope have proven difficult to scale, again partially as a result of complicated construction and procurement.

In response to the housing crisis and flat-lined and Prefabrication as an alternative. For example Design for Manufacturing and Assembly (DfMA) and modular timber construction aim to take as much labour off site
as possible. However, these approaches can't radically

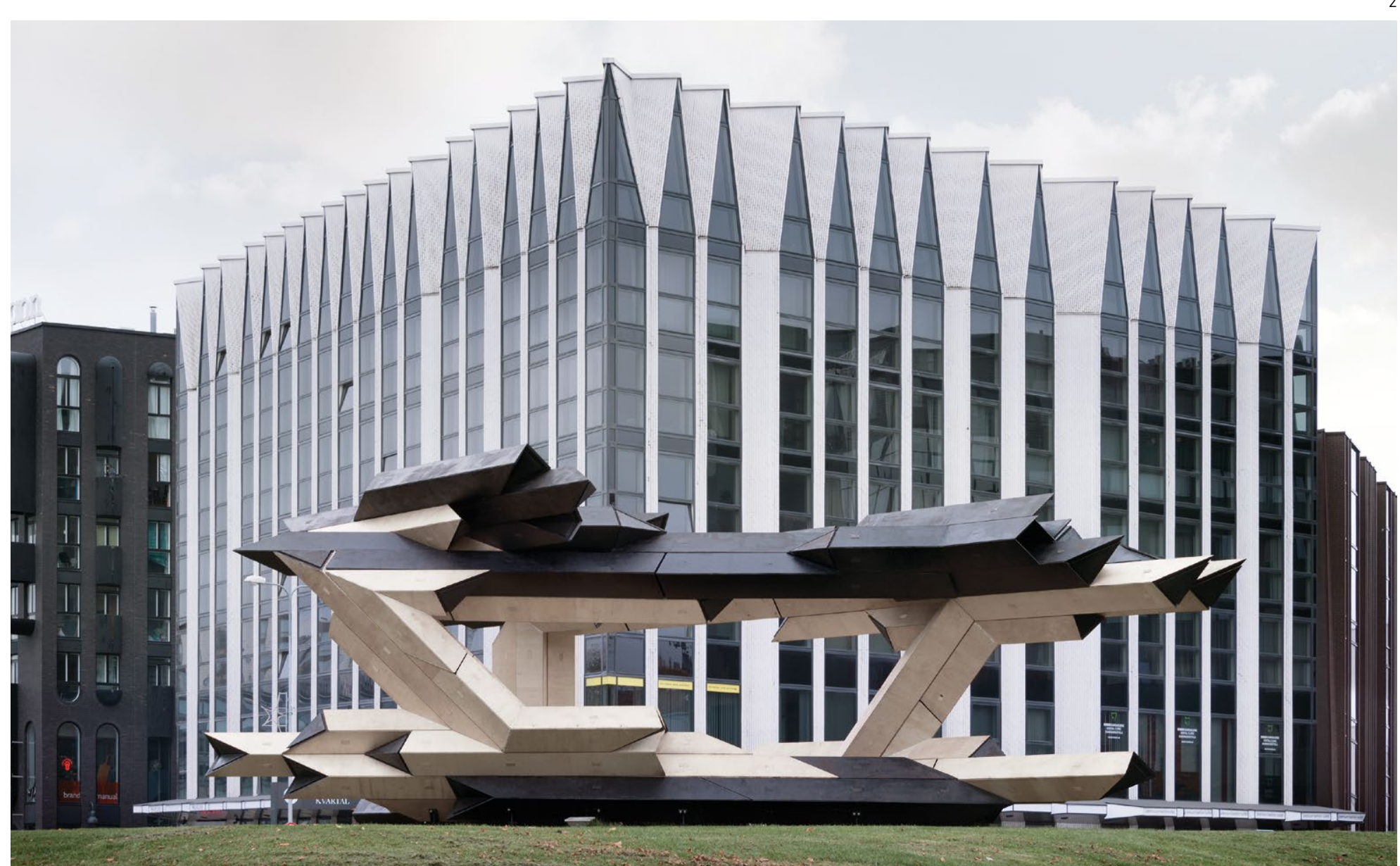

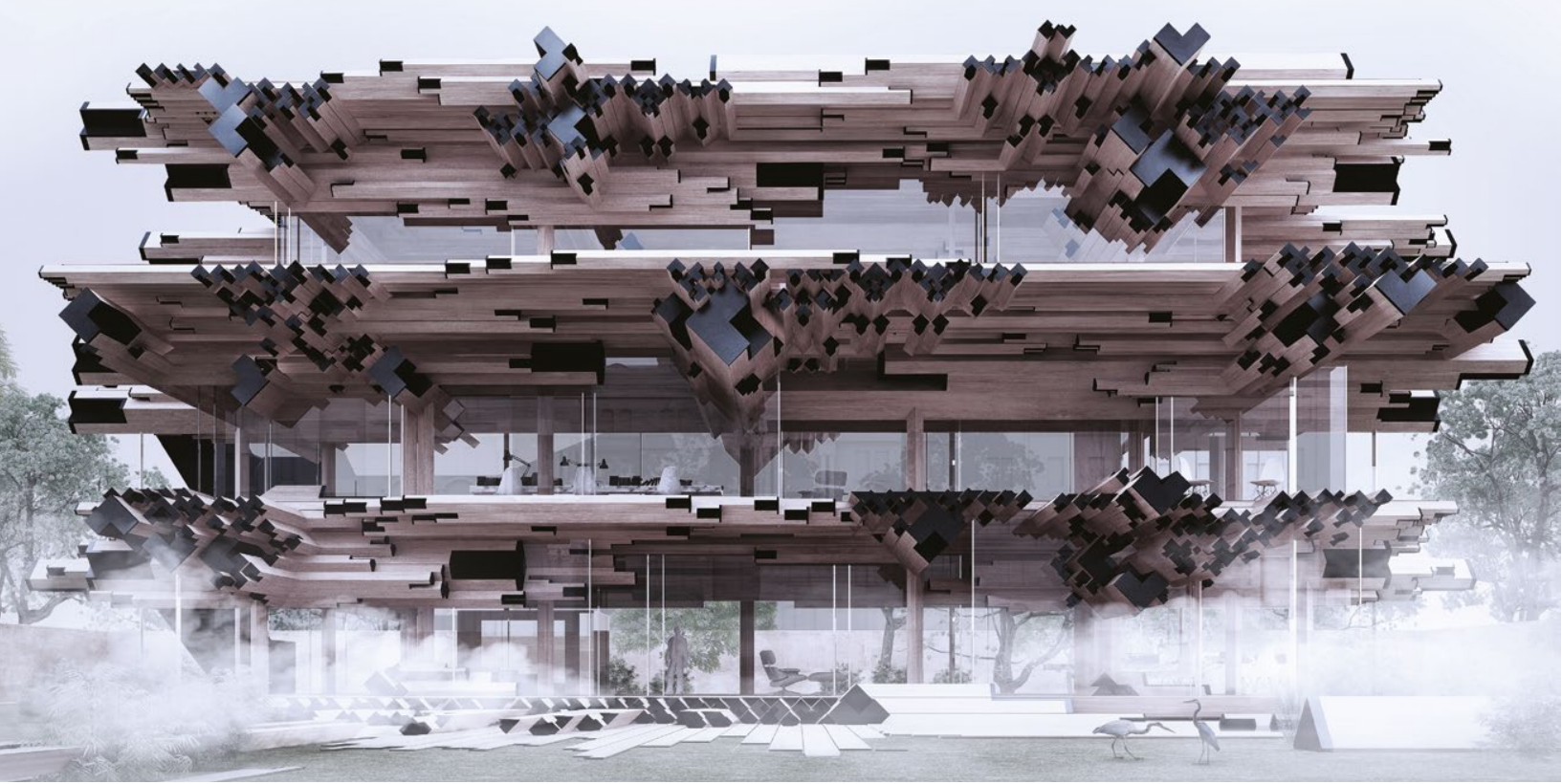

improve productivity as the production chain is still Moreover, the associated business model is reactive and service-based. Modules are one-offs that have to
be redesigned for every site and every project.

An approach based on a digital understanding of parts of Alfred Bemis and Leonardo Mosso present a historic precedent of a voxel-based architecture (Botazzi, 2018), as well as Frank Lloyd Wright's Textile Block houses. the Universal House as a discrete voxel-based building system (Morel, 2011), while Jose Sanchez' Polyomino project proposed discrete building blocks as plattorms for collaborative design (Sanchez, 2018). The interest in this work on Discreteness and Computation by Mario Carpo (Carpo, 2014).

Digital Modularity

This research attempts to transfer some of the coreestablishing a short, integrated, continuous production chain based on generic, serialised versatile building blocks cut from two-dimensional sheet materials, with limited connection possibilities and passive error unction is only established after assembly. The parts therefore exist independent of an actual building and all of life is assembled from just 20 standardised amino acids as modular building blocks (Langford, 2019).

The notion of digital fabrication is then redefined as the functional sure. An example of an actual digital Hiller's and Hod Lipson's proposal for 3D Voxel Printing, where physical voxels are defined as 'physical, selfaligning, fundamental units' (Hiller and Lipson, 2009) that are assembled by a printer. Can the construction of 政 process allows for a high degree of automation, both in the into buildings.

This research further questions a cost-effective, scalable method of production, the scale of the building blocks
themselves and the amount of variation within sets of elements. The large-scale, 1: prototypes aim to test the use of two dibilities ansiona bave mateh al, limited connection

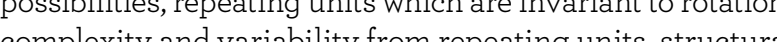
complexity and variability from repeating units, structural
capabilities, tension joints and the potential for automation. Tallinn Architecture Biennale Installation The question of an organic assembly of serialised, digitalwith the Diamonds House, a 2015 project for a multififamily dwelling in Belgium by Gilles Retsin Architecture (Fig. 3).
In collaboration with the UCL Design Computation Lab assembly or compilation of these building blocks into prefabrication of the parts and in the assembly of the parts 


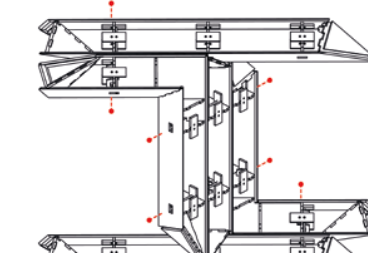

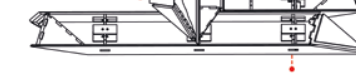
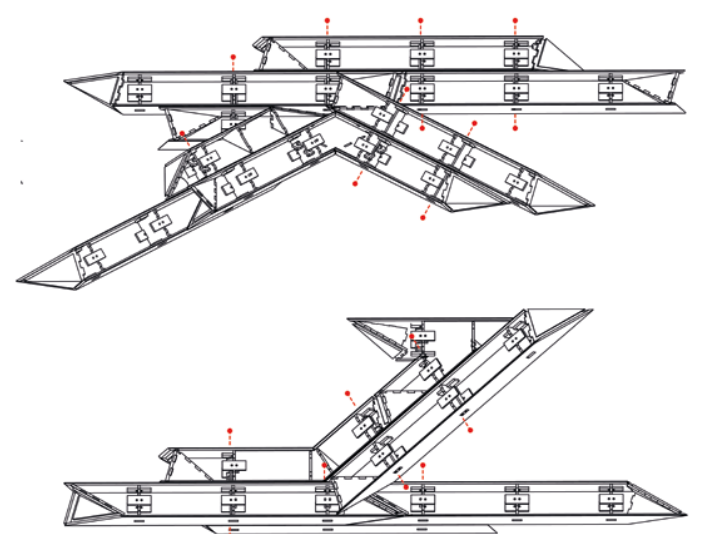

(DCI), an installation for the Tallinn Architecture Biennale test this idea on a 11.1 scale. The installation is a fragment of an abstract larger housing block, a fragment of which was then selected and further detailed (Figs 1, 2).

A family of discrete building blocks was developed: a straight element, a $90^{\circ}$ corner element, a $45^{\circ}$ corner
element and its inverse a 135 -degree corner element The straight element's proportions are derived from one sheet of exterior plywood (3300 x 1350 x 18mm). CNC machine using a standard and $45^{\circ}$ drill bit The toolpath was kept as simple as possible, to allow for quick cutting. The decision was made to avoid any visible finger joints or notches, details typical for CNC plywood projects. While there are internal notches, the sides of the and a series of $30 \mathrm{~mm}$ nails. The part is designed as a box beam-like element, consistent of a skin and three interior frames to stiffen the box. The interior frames are notched in the skin and hold a small plate with two $14 \mathrm{~mm}$-diame threaded rods (M10) which connect the parts laterllye The rods are fixed with polymer-insert lock nuts, to resist turning which could result from vibrations during the assembly. The internal stiffening frame coincides with the
position of the rods, forming a continuous stiffening fram

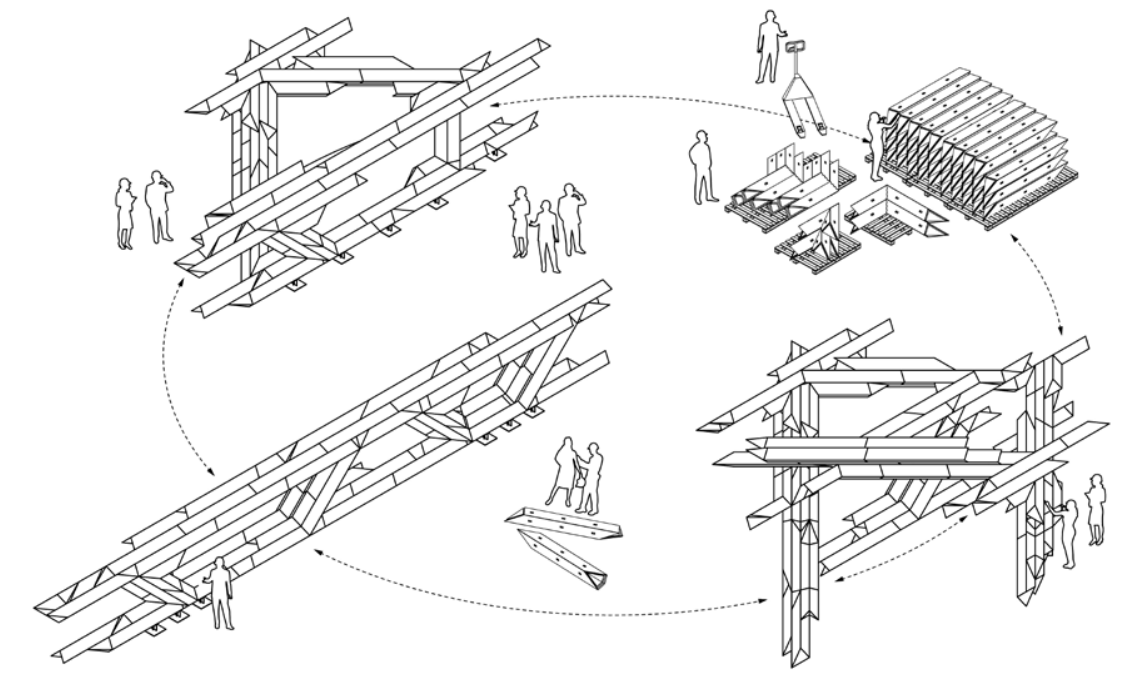

throughout multiple elements. These stiffening frames set out the modular rhythm and limited connection for the installation is defined by the $4 \mathrm{~mm}$ difference between the circular opening for the steel rods and the rod diameter. A $36 \mathrm{~mm}$-thick shear-key element is inserted in the opening lett for the rods

The male-female endings of the building blocks have no meds and cal connectivity, but together with the tension rods and stif thing fralles allow for passive enor correction orientation of the $45^{\circ}$ element a substantial amount of geometric interlocking is achieved. The different corner elements follow a similar build-up, but also incorporate an additional internal stiffening frame, a continuous piec This frame allows load-paths to shift and establishes uctural continuity (Fig. 4).

All elements are flip-invariant and appear multiple 6.Gilles Retinin. Tallin
Architecture Biennale the combination of steel rods and shear keys combines PAARA. 2017. Photo: the initially discrete building elements in a continuous monolithic structure

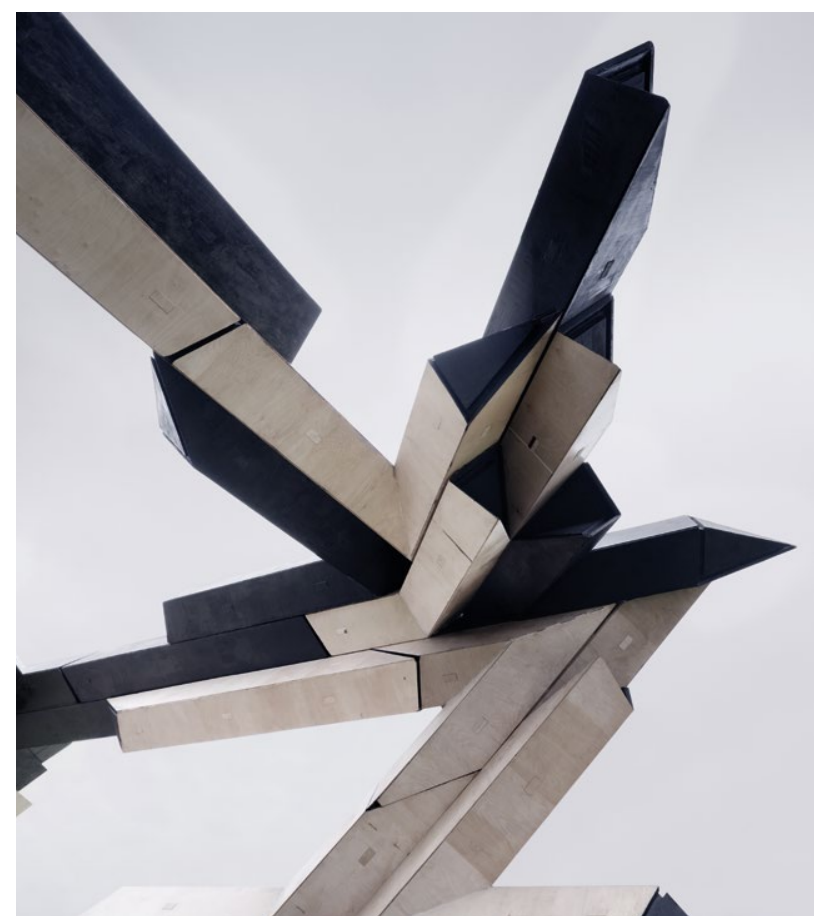

Cost-efficiency, variation and complexity

The TAB installation negotiates the challenges of complex, variegated large-scale structures out of serialised, function-agnostic, discrete building blocks. It presents a method for building discrete parts from a
single two-dimensional base material using only machine. The installation suffered minimal only one and deformations and was completed with an overall tolerance of $4 \mathrm{~mm}$ along a maximum length of $12.5 \mathrm{~m}$. Large cantilevers of up to $4.5 \mathrm{~m}$ were achieved, without digital detection ( H. t. 5 . The biggest difference with space-filling polyhedra or sphere as in most examples of digital materials, but are asymmetrical. The decision for a beam-like element has certain advantages on a large architectural scale: it allows for staggering and
overlapping and it reduces the number of joints.

The seriality of the elements and their properties of self-alignment and error correction allowed for a quick and efficient assembly, with only a small crew of four people in a space of five days - including site-prep
and finishing. Automation of on-site assembly is a long-term possibility, but doesn't appear as an absolue
necessity. If the scale of the parts is large enough, pre-assembly with a simple crane could be more

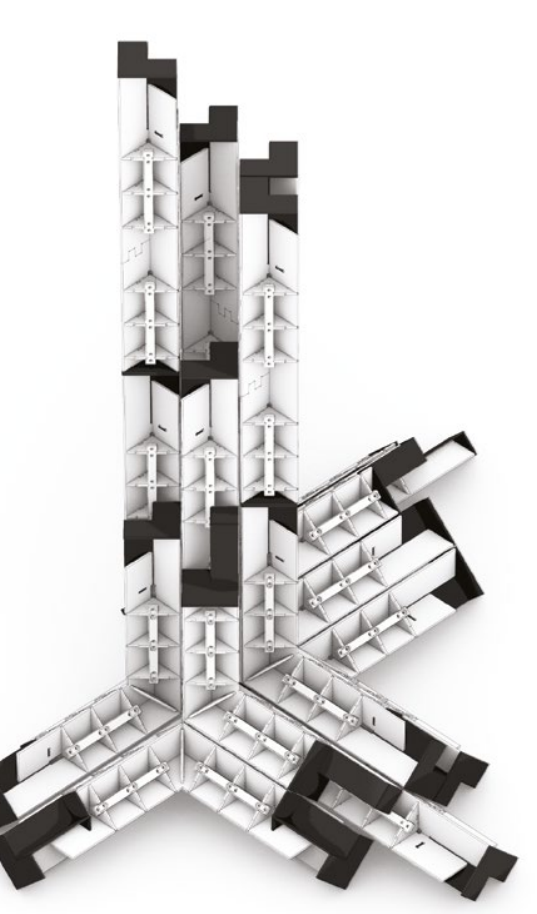

It was decided to deviate from the design model and move one element on Another installation at the Royal Academy (2019) questions the structural topology of the building block designed a s af mm plywood internal siff. Pants are designed as a $12 \mathrm{~mm}$ plywood internal stiffening frame in
three directions, connected to a light external skin of $9 \mathrm{~mm}$ plywood (Fig. 7). This significantly reduced the weight of the elements. A Hololens was used to stream instruction In both cases, the lack of mechanical connection in the male and female endings of the elements is problematic corner-elements to transfer forces between different planes. While the corner elements are efficient and of A subsequent installation developed for Tongji University in Shanghai (2019) introduces this connection, reducing the total number of elements to just one. If only one element is used, it's worth investing in robotically

7. Gilles Retsin,
Real Virtuality, Royal
Academy of Atrs, 2019 .
This was tested with the project ALIS, developed in the context of the Architectural Design MArch (B-Pro) 


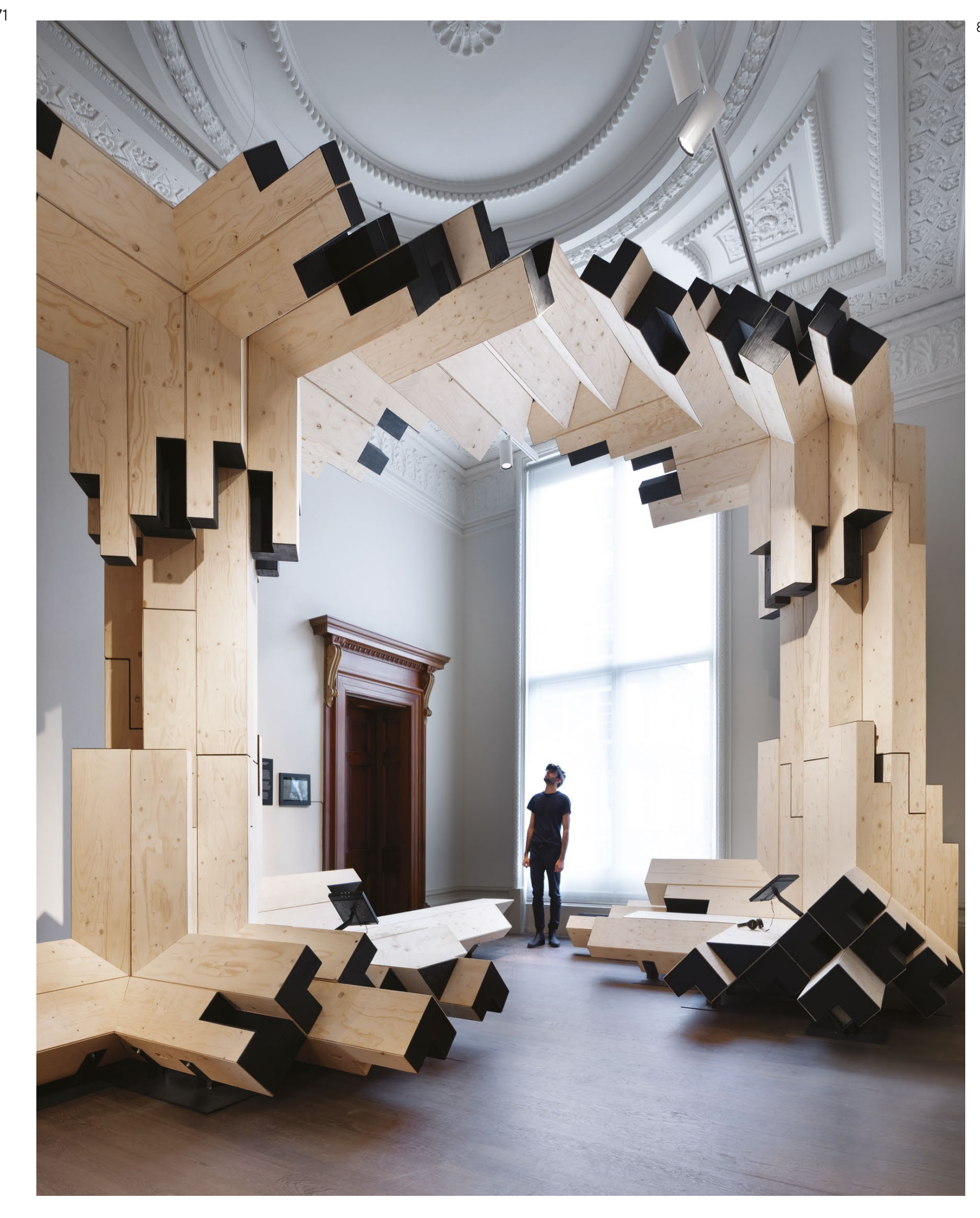

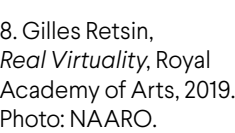

Master's students Joana Correia, Evgenia Krassakopoul ou,
Akhmet Khakimov, Kevin Saey and Estefania Barrios. Two industrial robots were used to pre-assemble a boxlike element. The robots pick and place pre-cut material from a preal stact on could be further improved with the use of press-fit joi as demonstrated by the research of Christopher Robelle et al. (2017)

Towards discrete automation

This research reveals a new notion of the architectural part as a discrete building block, combining aspects of digtal materialand program coble matter with architectural prefabication and modularity. Discrete falling construction productivity, the housing crisis and global climate crisis.

Once discretised, construction is more integrated and assembly becomes a continuous and organic process, and types commonly associated with assembly disappear. Physical and digital reality overlaps completely, there is no more representational gap between both. What is return computes This method therefore enables increased automation of construction, requiring potentially no minimal handling off site and only minimal manual handling on site.

By using timber, we make optimal use of large-scale small-scale manufacturing for the customisation and assembly of building elements and buildings. Each building could be assembled diferently, to a granula happen at no extra cost as the customised placement of elements is merely an informational task. Compared to modernist and current modular prefabrication, the function-agnostic, serial ised parts demonstrate increased based on sheet-materials is in turn agile and easy to customise to different building blocks. It does not prop a centrally controlled, universal, objective building block as a single solution.

The small-scale infrastructure needed to construct building more accessible, faster and therefore less capital intensive, opening the market to a larger group of house-builders.
Housing could be assembled, disassembled and adapted much faster, which in turn puts into question modes of forms of domesticity and procurement. 列 continuously recycled into other buildings.

As a further outlook, building blocks could become 'smart bricks', integrating mechanical functionality such as the transfer of heat, air, water or electricity. These could then be continuously assembled the filly thesenal buildings decentralised. Ultimately, the aspects presented here are the easiest part of construction, most labour is consumed on the fit-out of technical devices. However, it's with this Initial abstraction hat automation begins. Whithout firs automate is futile.

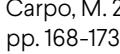

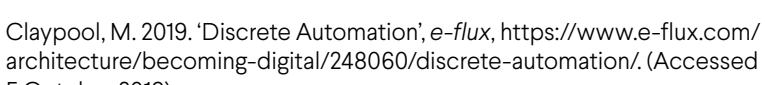
Cheung, K. 2012 . Digital
PhD Thesis, MIT, Bosto

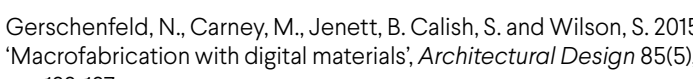
Langford, W. 2019. 'Discrete Robotic Construction'. PhD Thesis, MIT, Boston.

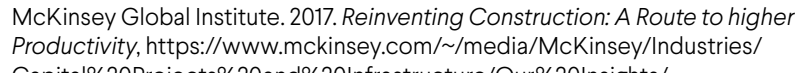

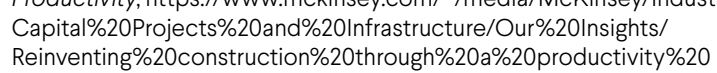

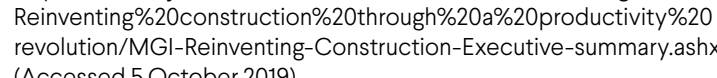

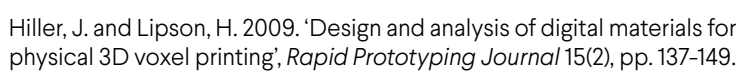

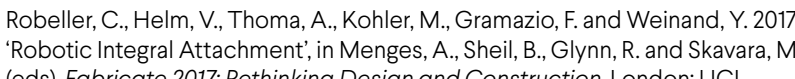
Press, pp.92-97. Morel, P. 2011. 'Sense and Sensibilia', Architectural Design 81414).pp. 122-129.

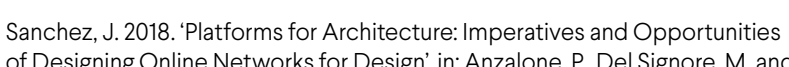

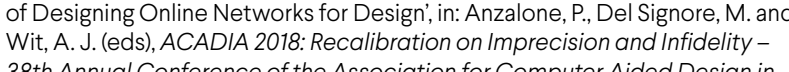

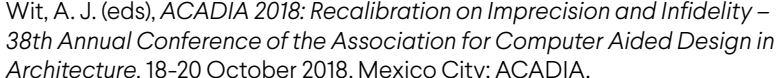

